\title{
Symmetry-constrained electron vortex propagation
}

\author{
L. Clark, ${ }^{1, *}$ G. Guzzinati, ${ }^{1}$ A. Béché, ${ }^{1}$ A. Lubk, ${ }^{2}$ and J. Verbeeck ${ }^{1}$ \\ ${ }^{1}$ EMAT, University of Antwerp, Groenenborgerlaan 171, 2020 Antwerp, Belgium \\ ${ }^{2}$ Triebenberglabor, University of Dresden, Zum Triebenberg 1, 01062 Dresden, Germany
}

(Received 4 March 2016; published 22 June 2016)

\begin{abstract}
Electron vortex beams hold great promise for development in transmission electron microscopy but have yet to be widely adopted. This is partly due to the complex set of interactions that occur between a beam carrying orbital angular momentum (OAM) and a sample. Herein, the system is simplified to focus on the interaction between geometrical symmetries, OAM, and topology. We present multiple simulations alongside experimental data to study the behavior of a variety of electron vortex beams after interacting with apertures of different symmetries and investigate the effect on their OAM and vortex structure, both in the far field and under free-space propagation.
\end{abstract}

DOI: 10.1103/PhysRevA.93.063840

\section{INTRODUCTION}

Electron vortex beams are electron states wherein the phase is structured to possess a phase singularity on-axis, with a wave function of $\Psi=A(r) e^{i \ell \phi} e^{i k z}$. The amplitude function in this simple case is a function of $r$ alone. The paraxial beam propagates in the $z$ direction, with wave number $k$. The value $\ell$ is an integer, describing the topological charge or winding number of the vortex. When the beam is in a simple, circularly symmetric state, $\ell$ also determines the orbital angular momentum (OAM) of the beam [1].

Such electron beams were first considered explicitly in 2007 [2], having been alluded to previously [3,4]. They were demonstrated experimentally in 2010 [5-7] and have remained under intense study since. The interest in these structured beams comes not only from a fundamental interest in their unusual behaviours [8-15], but also from prospects for novel applications in a variety of topics. Potential applications include magnetic chiral dichroism measurements [6,16-18], being used to determine crystal chirality [19] and providing opportunities to use electron vortices as an analog of optical tweezers [20].

The application most fervently awaited for electron vortex beams is that they may enable atomic-resolution electron magnetic circular dichroism (EMCD) $[6,16,18]$, with better resolution than existing methods. While early experiments seemed encouraging, later studies revealed the complexity of the setup, thus requiring further careful attention [21-23].

An electron beam interacts strongly with a sample [24], which rapidly changes the shape of the wave front. For typical TEM sample thicknesses, multiple scattering is likely to occur, further complicating the interaction between the beam electron and the sample. If the beam is aligned with a crystal zone axis of the sample, the interaction is particularly strong, and channeling will occur, biasing which parts of the sample are exposed to the probing electron [25]. This channeling will also affect the propagation of the vortex core $[26,27]$. The local symmetry imposed on the beam by the crystal structure will also affect the OAM of the wave as it propagates [28]. All of these effects occur synchronously,

*laura.clark@uantwerp.be leading to complicated details in the electron wave as it exits from the sample and complex changes in any recorded electron energy-loss spectrograms (as these depend on the local electron wave-function).

Here, we aim to clarify one aspect of this complex system, by studying the effects of spatial confinement in a geometric symmetry on an electron vortex beam. These are loosely analogous to the effects of the crystal symmetry on the electron vortex beam, while avoiding the complexity of channeling and other sample interaction effects.

Furthermore, in previous work we noted interesting behavior of electron vortex beams when microscope apertures are misaligned or when nonround apertures are used [26,29,30]. Particularly, following the interaction with apertures with discrete rotational symmetries reported by Guzzinati et al. [29], it can be seen that the original beam symmetry is not conserved, and a dark vortex core is not always found on-axis. The interference between the aperture edge waves [31] and the vortex probe leads to an intensity profile which reveals the topological charge of the input beam, in the case of a centered, single-ringed input vortex probe [32].

Such a non-circularly symmetric constraint prevents the beam from being in an OAM eigenstate, so a high-order vortex core may degenerate into an arrangement of multiple, displaced $|\ell|=1$ vortices [26]. We now deliberately apply these unusual settings to allow a deeper study of vortex splitting, propagation, and reconnection.

We investigate the vortex structure of an electron vortex beam after impinging on an aperture of a chosen geometry (round, square, equilateral triangle, off-centred circle), in the far field and during propagation. We study this system both computationally and experimentally. Similar systems have been considered previously with optical vortex beams, but only with regard to the far-field behavior of the beam. Here, we focus on the beam structure during propagation, as a route to a deeper understanding of electron vortices within a sample. In the following, we first compare the effects of the different geometric apertures on the far-field wave and vortex structures, comparing experimental data to our simulations. We then model the whole focal series of the beam, tracking the propagation of the vortex cores through the volume. We find a surprisingly complex vortex structure, which may be of significance to the development of the vortex-EMCD method. 


\section{SYSTEM DESCRIPTION}

Various methods of producing electron vortex beams have been investigated. The first experimental electron vortex beam relied on an opportune stacking of graphite flakes [5]. This is not easily reproducible. After that, the holographic forked mask technique, borrowed from optics [33], was introduced [6] and then developed further [7,34-37]. Electron vortices produced through aberration manipulation were also investigated [38-41], as well as direct phase plate production [42,43]. These methods, however, all have their own foibles-primarily, limitations to the intensity in a given vortex order or experimental limitations to the attainable purity of a given OAM state.

More recent developments, however, have improved on this. It has been shown that illuminating the end of a long, thin, nanoscale bar magnet can induce a vortex character into the electron beam, while only blocking a very small proportion of the incoming electron flux $[44,45]$. Experimentally, if the magnetic needle is produced sufficiently accurately, the resultant vortex beam can have more than $95 \%$ of the beam's intensity in the desired OAM state [46]. While this method results in the "best" electron vortex beams currently available, it is not well suited to investigation of different orders of topological charge, as this would require access to a set of several magnetic needle apertures. To ensure fairest comparison between vortex beams of different orders, we thus investigate the beams resulting from a well-made forked holographic mask, ensuring that they are sufficiently laterally separated. This provides the benefit of a high OAM-mode purity, at the expense of a lower beam intensity, not critical in the present study.

By using a forked holographic mask in the condenser plane of the microscope, we are able to produce a range of highorder vortex beams in the image plane, with diameters in the range of a few tens of nanometers (adjustable by tuning the condenser system). In the ideal, simple case, where the mask is designed by interfering a tilted plane wave with an $\exp (i \ell \phi)$ term only (with no additional amplitude modulation) and the binarization is applied at exactly half the maximum intensity value, the bars in the resulting mask will be the same width as the gaps [as illustrated in Fig. 1(a)]. In this case, the only beams produced will be the zero-order central beam (with $\ell=0$ ) and

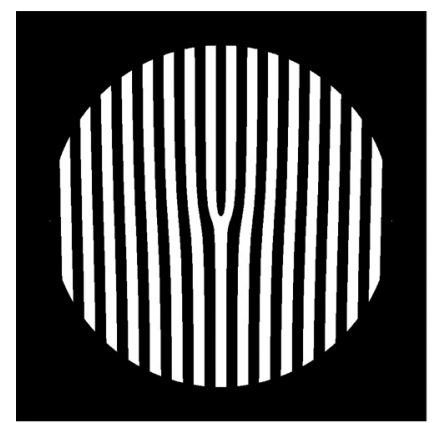

(a)

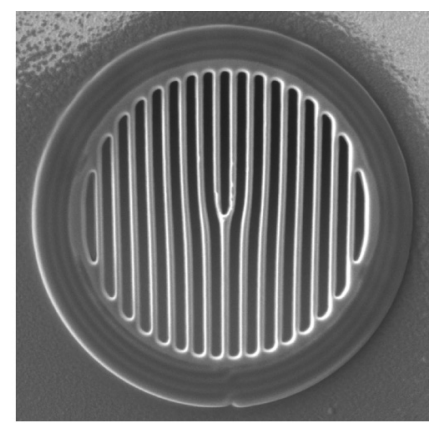

(b)
FIG. 1. Theoretical and experimental comparison of forked mask apertures. (a) Simple forked vortex mask design, with bar width identical to gap width. (b) Experimental forked mask, $10 \mu \mathrm{m}$ in diameter; note that the gaps are slightly wider than the bars. odd-ordered side beams (with $|\ell|=1,3,5, \ldots$ ), due to the combination of diffraction envelopes creating missing orders [47].

However, in many experimental cases, this does not hold true, due to the challenges in the fine control of the focusedion-beam milling time [35], leading to other vortex orders appearing, at varying intensities. The milling of our present experimental mask enables access to $|\ell|=(0,1,2,3)$ vortex orders with reasonable intensities.

For maximum diffractive effects, we want to produce the geometric apertures such that they are of a size similar to the diameter of the vortex beams in the sample plane. Due to the difficulties of producing apertures at this scale, the apertures in this work are implemented in the conjugate selected-area plane instead. With the illumination system of the microscope in probe-forming mode, our forked mask in the condenser plane (diameter, $10 \mu \mathrm{m}$; $\approx 0.25 \mathrm{mrad}$ ) produces vortex beams in the sample plane of around $30 \mathrm{~nm}$. In our microscope, there is a magnification factor of approximately 70 between the sample plane and the selected-area plane. This allows us to use apertures in the selected-area plane with a characteristic dimension of $2 \mu \mathrm{m}$. Thus, in the selected-area plane, the apertures and the vortex beams are of a similar relative size, leading to strong diffractive effects. We then defocus the beams slightly so as to optimally fill the apertures.

In this way, we can illuminate each of the geometric apertures with electron-vortex beams of different topological charges. We can image the far-field diffraction of each and compare these with simulations to investigate the resulting vortex structure. Furthermore, we can also manipulate the diffraction and projector lenses, such as to image a full series of the electron-vortex propagation volume, allowing us to study the free-space propagation of a pure vortex state after passage through a specific aperture. This will show how a pure state splits into a complicated combination of vortex states, depending on the symmetry of the system.

\section{A. Distinguishing topological charge and orbital angular momentum}

The terms OAM and topological charge are often used interchangeably. They do coincide in the well-studied case of a single, on-axis vortex beam. However, in the general case, they are different [48]. They can differ in magnitude, even in some on-axis, circularly symmetric cases [49], or differ in both sign and magnitude [50]. To aid clarity in the following sections, we explicitly define these concepts here.

Topological charge is identical to winding number and is a local property. It describes the number of times the phase passes through $2 \pi$ around a closed loop, $C$ :

$$
\ell=\frac{1}{2 \pi} \oint_{C} d \phi .
$$

If the loop itself passes through no zero-intensity points, $\ell$ is constrained to being an integer. The total topological charge in a region is only affected by the discrete points of phase discontinuity within that region. For an in-depth discussion on the nature of topological charge, see the work of Dennis [51].

The OAM is axis dependent, varying strongly depending on where in the beam it is measured from. It is conserved 
only in a circularly symmetric system [52]. Typically, in our paraxial systems, we are only interested in the $z$ component of the expectation value of the OAM,

$$
\left\langle L_{z}\right\rangle=\left\langle\Psi\left|\hat{L}_{z}\right| \Psi\right\rangle,
$$

where, in cylindrical coordinates, $\hat{L}_{z}=-i \hbar \frac{\partial}{\partial \hat{\theta}}$ [28,53]. In a system with discrete, rotational symmetry, a pure input state will broaden into a superposition of OAM modes upon propagation. The input vortex core may degenerate into a beam with multiple first-order vortices, displaced from the center of the incoming beam. Pairs of $(+1,-1)$ vortices can also be created, or annihilated within a freely propagating system, forming 3D vortex loops which do not change the global topology of the system [26,54].

\section{B. Endurance of geometrical symmetry}

In addition to the propagation behavior enforced by the conservation of topology, the standard behavior of Fourier optics must still apply. It is trivial to show that the intensity pattern in the far field of a plane wave limited by an aperture is the squared modulus of the Fourier transform of the wave in the aperture plane [55]. Distorting the input wave from a planar wave front to something else will lead to a convolution of the Fourier transform of the aperture shape with the Fourier transform of the input wave. Combining these effects means that the complete rotational symmetry of the incoming vortex beam is reduced to the discrete rotational symmetry of the aperture.

\section{FAR-FIELD BEHAVIOR}

We begin investigating these effects by studying far-field intensities of different vortex beam orders on a set of apertures with different rotational symmetries.

\section{A. Simulated results}

First, the far-field results of illuminating a series of geometrical apertures were simulated. The chosen apertures were a centered circle, an equilateral triangle, a square, and a circle displaced by half a radius. These were modeled as binary arrays. In each case the aperture was then multiplied by an array representing the appropriate vortex wave $[\Psi \propto \exp (i \ell \phi)]$. The resultant far-field wave was then calculated by two-dimensional discrete Fourier transform [55].

In Fig. 2, these apertures are illustrated, along with figures representing the intensity (brightness) and phase (hue) of the wave in the far field of the aperture when illuminated with vortex beams of different orders. The vortex beam was modelled as a beam resulting from the far-field diffraction of a forked holographic mask, with the size chosen such that the peak intensity ring of the vortex approximately matched the size of the aperture.

\section{B. Experimental results}

Corresponding experiments were then performed in an aberration-corrected FEI $\operatorname{Titan}^{3}$ microscope operating at $200 \mathrm{keV}$, resulting in the data illustrated in Fig. 3. The forked aperture was located in the condenser plane, with the illumination system operated in probe-forming mode, generating an array of vortex beams in the sample plane. The objective and diffraction lens reproduced this pattern magnified 70 times in the selected-area plane, impinging on the geometrical apertures. The projection system, operated in diffraction mode, projected the far-field intensity of the illuminated geometrical aperture onto the CCD.

Comparing the simulated and experimental far-field images, as shown in Figs. 2 and 3, we can see a very good agreement of the intensity profiles. The results begin to diverge in the $\ell=3$ data set, as these beams pass at a higher angle to the optical axis through the microscope from the forked mask and thus are more sensitive to the residual aberrations.

The trivial $\ell=0$ cases simply show the intensity of a Fourier-transformed approximately Gaussian beam, apodized by these apertures [47]. The $\ell=0$ triangle case begins to reveal the discrete symmetry. Friedel's law points out that the Fourier transform of a real function is centrosymmetric, and thus the intensity pattern of diffraction from a triangular aperture will have sixfold symmetry. However, when the conditions for Friedel's law are broken, such as by a nonreal or phase-structured incoming wave (such as in $|\ell| \geqslant 1$ cases), the diffraction pattern is no longer compelled to be centrosymmetric [19], and the diffraction pattern can then reveal more directional information about the probe. For example, Mourka et al. established that diffraction of an optical vortex beam from an aperture without $180^{\circ}$ rotational symmetry can determine its handedness, through interaction of the OAM-induced rotation with the Gouy phase [56,57]. It has also been shown, in both light optics and electron microscopy, that diffraction of a single-ringed vortex beam from a triangular aperture enables quantification of the topological order of the vortex beam by counting the $|\ell|+1$ intensity lobes found along one edge of the resultant triangular pattern of lobes [29,32]. A closer look at the intensity profile of the $\ell=3$ triangle case in Fig. 3 reveals a faint-intensity lobe on-axis, even in the idealized simulation in Fig. 2. This is unusual in coherent vortex studies and warrants further attention. A related unusual effect can be seen in the $\ell=3$ square case, where, in the center of the intensity profile, five dark spots can be seen, arranged as on a die.

To investigate the cause of these effects, the simulations were further developed to highlight the vortex cores and determine their handedness. Vortex cores were located by finding peaks in the curl of the wave vectors (wave-vector vorticity) [48] in each propagated plane. However, in a small, pixelated loop around a vortex core, phase aliasing errors can quickly creep in when dealing with higher order vortices (with their rapid phase changes). To avoid this complication, images shown in Fig. 4 differentiate only between the left- and the right-handedness of the vortices, and not between their topological orders.

In the first column in Fig. 4, we see the standard behavior of the unperturbed beam; the single vortex core of each beam remains stable and on-axis, as the cylindrical symmetry permits OAM eigenstates [58]. There are phase jumps of $\pi$ between the rings in the tails of the Airy disk, but these do not affect the vortex behavior in this cylindrically symmetric case.

However, in the triangle and square cases (second and third columns in Fig. 4) vortices are abundantly present in the $\ell=0$ far-field profiles. This is due to the interference between the 


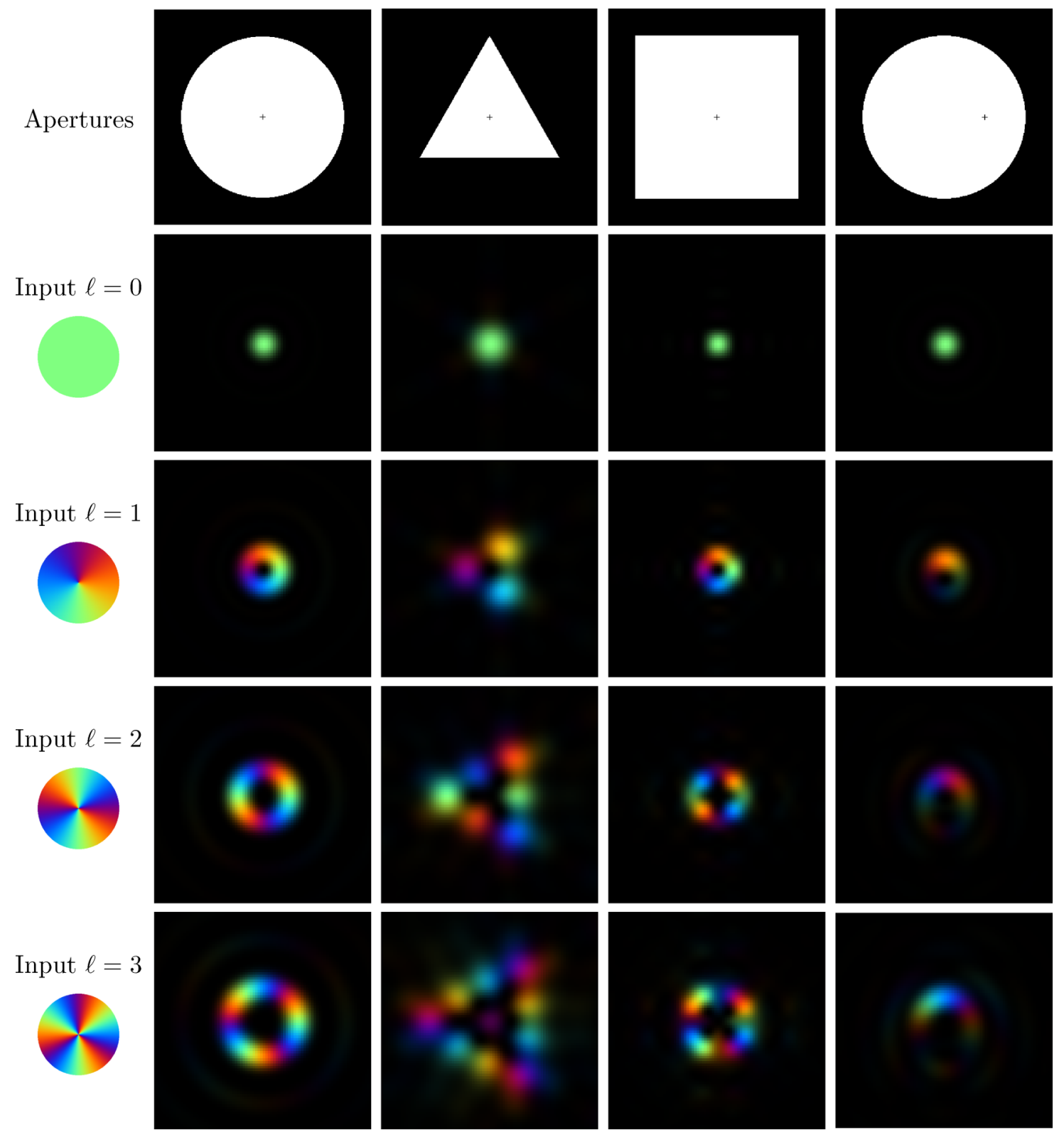

FIG. 2. Simulated apertures and far-field intensity patterns resulting from vortex beams of different orders. The $0-2 \pi$ phase color map is illustrated in the $\ell=1$ incoming vortex probe. The + marker in each aperture highlights the center of the incoming vortex beam. Apertures have edge lengths, or diameters, of $2 \mu \mathrm{m}$. The phase of the wave front is represented by the hue of the wave, as indicated in the leftmost column.

different sets of Fresnel fringes propagating from the aperture sides [31] in such a way as to produce arrays of $(+1,-1)$ vortices, similarly to the vortex lattices produced by Niermann et al. [59].

In addition to the many vortex-antivortex pairs created in the outer region of the pattern, in some of the higher order cases, we can also note interesting splitting behaviors of the central vortex. The splitting occurs only when the order of the vortex is greater than half the order of the rotational symmetry of the aperture; this inequality was first noted by Ferrando [52]. We see evidence of this in the $\ell=2$, square aperture case. It is unusual for a high-order vortex not to degenerate in a non-circularly symmetric system, but as in this case, $|m| \leqslant \frac{n}{2}$, following the notation of García-March et al., where integer $m$ is the angular momentum of the input beam and $n$ is the order of rotational symmetry of the system [60]. In the case where the vortex order matches the geometrical, rotational symmetry of the aperture, such as in the $\ell=3$ beam in a triangle, we see that the vortex splits into $\ell$ distinct vortices of order 1 . In cases where the vortex order does not match the aperture symmetry but does surpass Ferrando's inequality, such that the vortex core must degenerate, additional $(+1,-1)$ vortex pairs can be created such that both topological and geometrical requirements are fulfilled. We see this effect clearly in the $\ell=2$ triangle case and in the $\ell=3$ square case, where, in the central region of the diffraction pattern, we can see extra vortex cores in Fig. 4. The off-center circle, however, shows a rather different vortex structure, due to the complete absence of rotational symmetry in the system. In this case, higher order vortices split without the creation of additional vortices in 


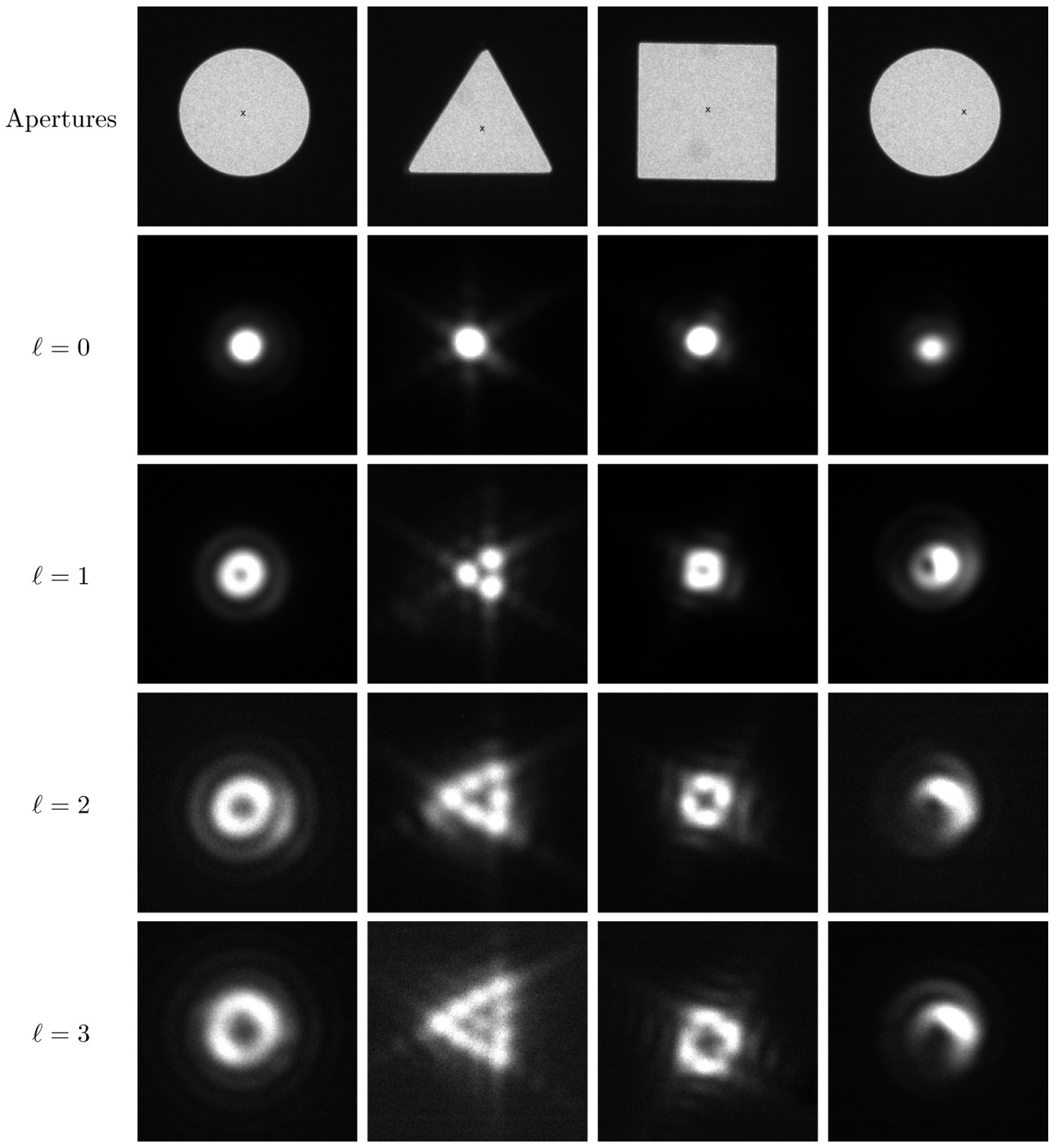

FIG. 3. Experimental apertures and far-field intensity patterns resulting from vortex beams of different orders. The $\mathrm{X}$ marker in each aperture highlights the center of the incoming vortex beam. Apertures have edge lengths, or diameters, of $2 \mu \mathrm{m}$.

the central region of the diffraction pattern. There are many $(+1,-1)$ vortex pairs in the surrounding area, particularly along a line perpendicular to the aperture-shift direction (half of the Gouy rotation for a full image-to-image propagation [61]). The rotations seen in the experimental case do not match this, due to the experimental difficulties of positioning the aperture. We note the interesting parallel between this infinite line of vortices and the vortices resulting from a fractional phase step [62].

\section{Propagation series}

The far-field structures certainly have interesting and unusual structures, which are structurally very different from the input wave fronts. For this reason, we decided also to investigate the wave-front structure upon propagation.
A simulation was built around a thin-lens model, propagating from the image plane (showing the aperture outline) towards the back-focal plane (containing the far-field image). This range is too large to be numerically modeled with a simple Fresnel propagator without encountering the sampling issues common in such propagation methods [63]. A modified linear canonical transform algorithm was used instead, wherein the fractional Fourier transform allows us to sample the full range of the propagation volume. The modeled system is an $\ell=3$ Laguerre-Gaussian vortex beam, illuminating a square aperture. This example was selected as it displays most of the interesting features in one system.

Experimentally, this was performed at $300 \mathrm{keV}$, with the same microscope setup as described in Sec. III B. The focal series is obtained by defocusing from the far-field intensity pattern towards an image of the aperture. This defocusing, 


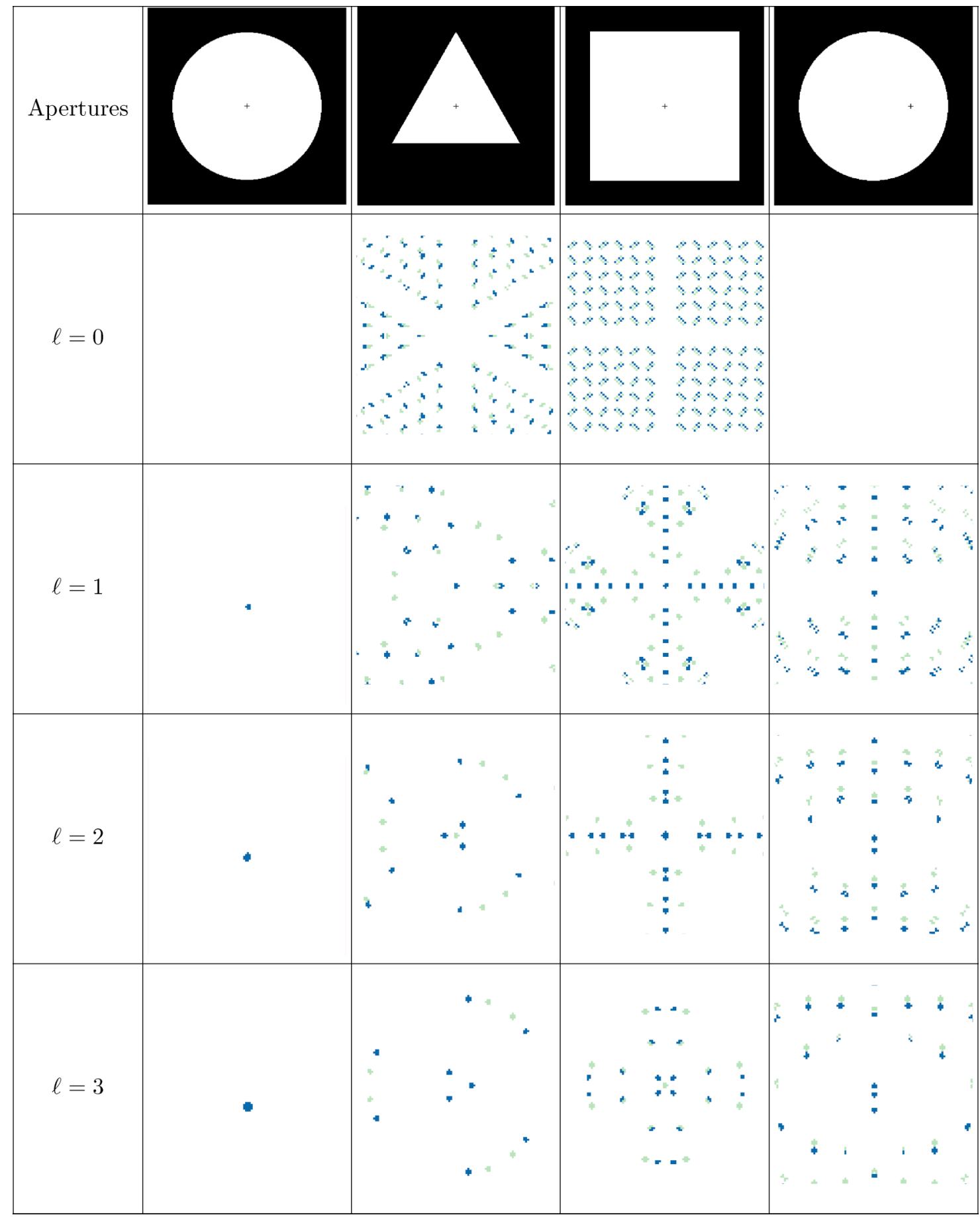

FIG. 4. Simulated apertures and far-field vortex patterns resulting from vortex beams of different orders. The + marker in each aperture highlights the center of the incoming vortex beam. Blue symbols correspond to a positive (right-handed) vortex core, while green symbols correspond to a negative (left-handed) vortex core.

however, changes the currents in the diffraction lens, such that the principal planes and relative position become undefined. Subsequently, the magnification of the system changes nonlinearly with the defocus. For this reason, the experimental focal series data are unscaled and can be compared with the simulations only qualitatively. Accordingly, the simulations are unscaled in the $(x, y)$ plane.

The simulation and experiment match closely, as can be seen in sample images from each focal series, displayed in
Fig. 5. From the simulated series, we are also able to locate the vortex cores (using the same algorithm as for Fig. 4) in each $(x, y)$ plane. These can then be collated over the series in $z$ by tracking isosurfaces enclosing volumes of high vorticity. This is shown in Fig. 6.

In this figure, we can see several regimes, as highlighted by the annotations. The incoming single vortex at the bottom of the figure is initially stable. The vortex core then splits into a complex lattice of vortices; the requirement of at least 

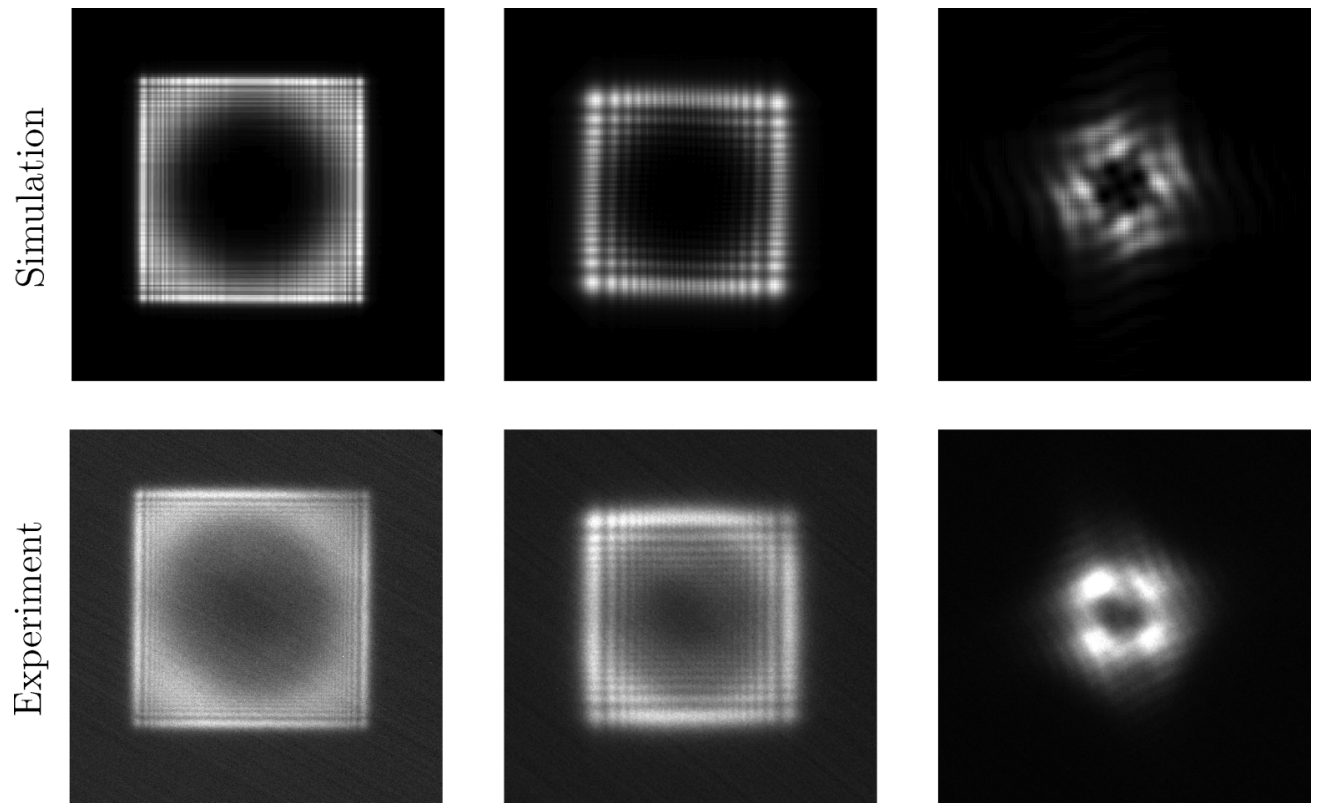

FIG. 5. Propagation series of an $\ell=3$ vortex beam after a square aperture, from the near field (left) towards the far field (right). The upper row shows simulated data (perfect coherence); the lower row, experimental data.

three-plane-wave interference for a vortex lattice is met, with edge waves meeting the paraxial beam [64], leading to the initial vortex bifurcations. Looking farther from the aperture in $z$, the created vortices annihilate in $(+1,-1)$ pairs. This is possible due to the presence of more than three plane waves [65]. The system cannot be expressed in only a few plane-wave terms, and hence the output is very complex. The periodicity of the vortex creation-annihilation loops decreases with the frequency of the Fresnel fringes under propagation, until the far field is reached, transitioning approximately as the Fresnel number approaches $F=1$.

As the system reaches the far field, the rules defined by Ferrando and García-March express themselves more clearly [66], with the $\langle\ell\rangle=3$ form of the beam in a square symmetry, composed of four $\ell=+1$ and one $\ell=-1$ vortex cores in

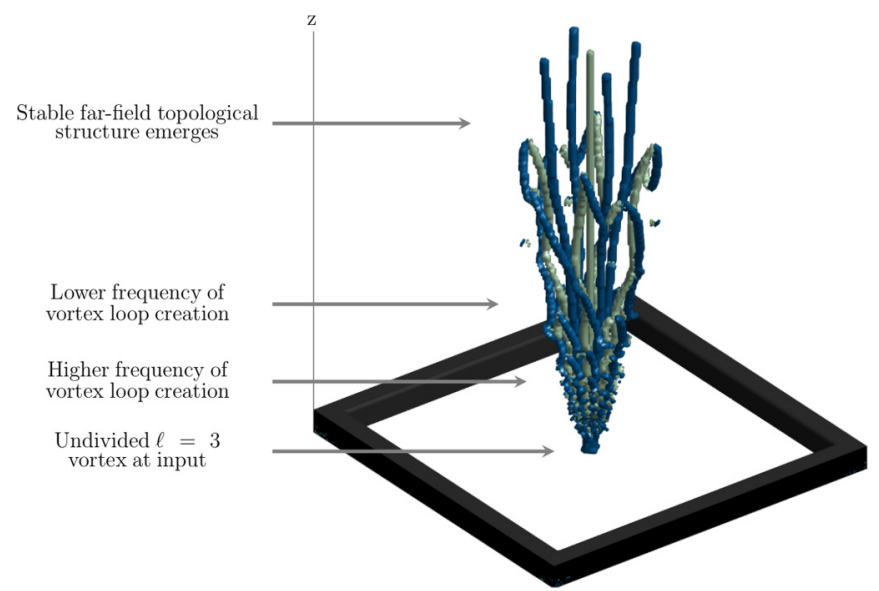

FIG. 6. Tracking of the vortex cores of an $\ell=3$ vortex core, illuminating a square aperture to the far field. Blue symbols correspond to a positive (right-handed) vortex core, while green symbols correspond to a negative (left-handed) vortex core. the central region. In this diffraction pattern, around $86 \%$ of the wave front is found to still be in an $\ell=3$ state (measured over the whole wave, on-axis, using the decomposition method employed in Ref. [39]).

Unlike the model system of $\mathrm{O}$ 'Holleran et al., we do not see any interlinked, closed loops [67]. This is probably due to the predominant $z$-only momentum of the system. Unlike Dennis et al., we do not see any knots (excluding the unknot) [68], due to the nongeneric aperture shapes required to produce a knot.

\section{CONCLUSION}

We have studied the propagation of vortex cores in electron vortex beams in non-rotationally symmetric systems. High-order vortex cores were shown to split, and it is demonstrated that a stable far-field pattern can include more vortex-antivortex pairs than were present in the initial condition. The propagation volume leading to such patterns was investigated for the first time, and its complexity was studied. The vortex structure under near-field conditions was found to be exceptionally detailed.

The instability of vortices and the ease with which they can be created under propagation are significant contributing factors to the challenges of using electron vortex beams to reproducibly measure the magnetic states of a material using electron energy-loss spectroscopy (EELS). The results presented in this paper suggest that to optimize vortex-EMCD, the effect of the lattice symmetry on the vortex beam needs to be minimized, perhaps through careful selection of the beam convergence angle and channeling conditions.

\section{ACKNOWLEDGMENTS}

L.C., A.B., G.G., and J.V. acknowledge funding from the European Research Council under the 7th Framework Program 
(FP7), ERC Starting Grant No. 278510_VORTEX. J.V. and A.L. acknowledge financial support from the European Union through the 7th Framework Program (FP7) under a contract for an Integrated Infrastructure Initiative (Reference No. 312483 ESTEEM2). The Qu-Ant-EM microscope was partly funded by the Hercules fund of the Flemish Government.
[1] L. Allen, M. W. Beijersbergen, R. J. C. Spreeuw, and J. P. Woerdman, Orbital angular momentum of light and the transformation of Laguerre-Gaussian laser modes, Phys. Rev. A 45, 8185 (1992).

[2] K. Yu. Bliokh, Y. P. Bliokh, S. Savel'ev, and F. Nori, Semiclassical Dynamics of Electron Wave Packet States with Phase Vortices, Phys. Rev. Lett. 99, 190404 (2007).

[3] I. Bialynicki-Birula, Z. Bialynicka-Birula, and C. Śliwa, Motion of vortex lines in quantum mechanics, Phys. Rev. A 61, 032110 (2000).

[4] A. Fukuhara, K. Shinagawa, A. Tonomura, and H. Fujiwara, Electron holography and magnetic specimens, Phys. Rev. B 27, 1839 (1983).

[5] M. Uchida and A. Tonomura, Generation of electron beams carrying orbital angular momentum, Nature 464, 737 (2010).

[6] J. Verbeeck, H. Tian, and P. Schattschneider, Production and application of electron vortex beams, Nature 467, 301 (2010).

[7] B. J. McMorran, A. Agrawal, I. M. Anderson, A. A Herzing, H. J Lezec, J. J. McClelland, and J. Unguris, Electron vortex beams with high quanta of orbital angular momentum, Science 331, 192 (2011).

[8] R. Van Boxem, J. Verbeeck, and B. Partoens, Spin effects in electron vortex states, Europhys. Lett. 102, 40010 (2013).

[9] R. Van Boxem, B. Partoens, and J. Verbeeck, Rutherford scattering of electron vortices, Phys. Rev. A 89, 032715 (2014).

[10] R. Van Boxem, B. Partoens, and J. Verbeeck, Inelastic electronvortex-beam scattering, Phys. Rev. A 91, 032703 (2015).

[11] S. M. Lloyd, M. Babiker, and J. Yuan, Interaction of electron vortices and optical vortices with matter and processes of orbital angular momentum exchange, Phys. Rev. A 86, 023816 (2012).

[12] S. M. Lloyd, M. Babiker, J. Yuan, and C. Kerr-Edwards, Electromagnetic Vortex Fields, Spin, and Spin-Orbit Interactions in Electron Vortices, Phys. Rev. Lett. 109, 254801 (2012).

[13] K. Y. Bliokh, M. R. Dennis, and F. Nori, Relativistic Electron Vortex Beams: Angular Momentum and Spin-Orbit Interaction, Phys. Rev. Lett. 107, 174802 (2011).

[14] B. G. Mendis, Dynamic scattering of electron vortex beams-A Bloch wave analysis, Ultramicroscopy 149, 74 (2015).

[15] Y. Hasegawa, K. Saitoh, N. Tanaka, S. Tanimura, and M. Uchida, Young's interference experiment with electron beams carrying orbital angular momentum, J. Phys. Soc. Jpn. 82, 033002 (2013).

[16] P. Schattschneider, S. Löffler, M. Stöger-Pollach, and J. Verbeeck, Is magnetic chiral dichroism feasible with electron vortices? Ultramicroscopy 136, 81 (2014).

[17] J. Verbeeck, P. Schattschneider, S. Lazar, M. Stöger-Pollach, S. Löffler, A. Steiger-Thirsfeld, and G. Van Tendeloo, Atomic scale electron vortices for nanoresearch, Appl. Phys. Lett. 99, 203109 (2011).

[18] J. Rusz and S. Bhowmick, Boundaries for Efficient Use of Electron Vortex Beams to Measure Magnetic Properties, Phys. Rev. Lett. 111, 105504 (2013).

[19] R. Juchtmans, A. Béché, A. Abakumov, M. Batuk, and J. Verbeeck, Using electron vortex beams to determine chirality of crystals in transmission electron microscopy, Phys. Rev. B 91, 094112 (2015).

[20] J. Verbeeck, H. Tian, and G. Van Tendeloo, How to manipulate nanoparticles with an electron beam? Adv. Mater. 25, 1114 (2013).

[21] J. Rusz, S. Bhowmick, M. Eriksson, and N. Karlsson, Scattering of electron vortex beams on a magnetic crystal: Towards atomicresolution magnetic measurements, Phys. Rev. B 89, 134428 (2014).

[22] J. Rusz, J.-C. Idrobo, and S. Bhowmick, Achieving Atomic Resolution Magnetic Dichroism by Controlling the Phase Symmetry of an Electron Probe, Phys. Rev. Lett. 113, 145501 (2014).

[23] D. Pohl, S. Schneider, J. Rusz, and B. Rellinghaus, Electron vortex beams prepared by a spiral aperture with the goal to measure EMCd on ferromagnetic films via stem, Ultramicroscopy $\mathbf{1 5 0}$, 16 (2015).

[24] R. Henderson, The potential and limitations of neutrons, electrons and $\mathrm{x}$-rays for atomic resolution microscopy of unstained biological molecules, Q. Rev. Biophys. 28, 171 (1995).

[25] N. R. Lugg, G. Kothleitner, N. Shibata, and Y. Ikuhara, On the quantitativeness of EDS STEM, Ultramicroscopy 151, 150 (2015).

[26] A. Lubk, L. Clark, G. Guzzinati, and J. Verbeeck, Topological analysis of paraxially scattered electron vortex beams, Phys. Rev. A 87, 033834 (2013).

[27] S. Löffler and P. Schattschneider, Elastic propagation of fast electron vortices through crystals, Acta Crystallogr. Sec. A: Found. Crystallogr. 68, 443 (2012).

[28] G. Molina-Terriza, J. P. Torres, and L. Torner, Management of the Angular Momentum of Light: Preparation of Photons in Multidimensional Vector States of Angular Momentum, Phys. Rev. Lett. 88, 013601 (2001).

[29] G. Guzzinati, L. Clark, A. Béché, and J. Verbeeck, Measuring the orbital angular momentum of electron beams, Phys. Rev. A 89, 025803 (2014).

[30] L. Clark, A. Béché, G. Guzzinati, and J. Verbeeck, Quantitative measurement of orbital angular momentum in electron microscopy, Phys. Rev. A 89, 053818 (2014).

[31] J. B. Keller, Geometrical theory of diffraction, J. Opt. Soc. Am. 52, 116 (1962).

[32] J. M. Hickmann, E. J. S. Fonseca, W. C. Soares, and S. ChávezCerda, Unveiling a Truncated Optical Lattice Associated with a Triangular Aperture Using Light's Orbital Angular Momentum, Phys. Rev. Lett. 105, 053904 (2010).

[33] V. Yu. Bazhenov, M. V. Vasnetsov, and M. S. Soskin, Laser beams with screw dislocations in their wavefronts, JETP Lett. 52, 429 (1990).

[34] J. Verbeeck, H. Tian, and A. Béché, A new way of producing electron vortex probes for stem, Ultramicroscopy 113, 83 (2012).

[35] L. Clark, Electron Vortex Beams: Production and Propgation, Master's thesis, University of York, 2012. 
[36] V. Grillo, G. C. Gazzadi, E. Karimi, E. Mafakheri, R. W. Boyd, and S. Frabboni, Highly efficient electron vortex beams generated by nanofabricated phase holograms, Appl. Phys. Lett. 104, 043109 (2014).

[37] T. R. Harvey, J. S. Pierce, A. K. Agrawal, P. Ercius, M. Linck, and B. J. McMorran, Efficient diffractive phase optics for electrons, New J. Phys. 16, 093039 (2014).

[38] T. C. Petersen, M. Weyland, D. M. Paganin, T. P. Simula, S. A. Eastwood, and M. J. Morgan, Electron Vortex Production and Control Using Aberration Induced Diffraction Catastrophes, Phys. Rev. Lett. 110, 033901 (2013).

[39] L. Clark, A. Béché, G. Guzzinati, A. Lubk, M. Mazilu, R. Van Boxem, and J. Verbeeck, Exploiting Lens Aberrations to Create Electron-Vortex Beams, Phys. Rev. Lett. 111, 064801 (2013).

[40] P. Schattschneider, M. Stöger-Pollach, and J. Verbeeck, Novel Vortex Generator and Mode Converter for Electron Beams, Phys. Rev. Lett. 109, 084801 (2012).

[41] A. H. Tavabi, V. Migunov, C. Dwyer, R. E. Dunin-Borkowski, and G. Pozzi, Tunable caustic phenomena in electron wavefields, Ultramicroscopy 157, 57 (2015).

[42] R. Shiloh, Y. Lereah, Y. Lilach, and A. Arie, Sculpturing the electron wave function using nanoscale phase masks, Ultramicroscopy 144, 26 (2014).

[43] A. Béché, R. Winkler, H. Plank, F. Hofer, and J. Verbeeck, Focused electron beam induced deposition as a tool to create electron vortices, Micron 80, 34 (2016).

[44] A. Béché, R. Van Boxem, G. Van Tendeloo, and J. Verbeeck, Magnetic monopole field exposed by electrons, Nature Phys. 10, 26 (2014).

[45] A. M. Blackburn and J. C. Loudon, Vortex beam production and contrast enhancement from a magnetic spiral phase plate, Ultramicroscopy 136, 127 (2014).

[46] A. Béché, R. Juchtmans, and J. Verbeeck, Efficient creation of electron vortex beams for high resolution STEM imaging, Ultramicroscopy (2016).

[47] E. Hecht, Optics (Addison Wesley, Reading, MA, 1998).

[48] M. V. Berry, Optical currents, J. Opt. A: Pure Appl. Opt. 11, 094001 (2009).

[49] A. M. Amaral, E. L. Falcão-Filho, and C. B. de Araújo, Characterization of topological charge and orbital angular momentum of shaped optical vortices, Opt. Express 22, 30315 (2014).

[50] G. Molina-Terriza, Vortex transformations and vortex dynamics in optical fields, in The Angular Momentum of Light, edited by D. L. Andrews and M. Babiker (Cambridge University Press, Cambridge, UK, 2012), pp. 31-50.
[51] M. R. Dennis, Topological Singularities in Wave Fields, Ph.D. thesis, University of Bristol, 2001.

[52] A. Ferrando, Discrete-symmetry vortices as angular bloch modes, Phys. Rev. E 72, 036612 (2005).

[53] P. Schattschneider and J. Verbeeck, Theory of free electron vortices, Ultramicroscopy 111, 1461 (2011).

[54] L. J. Allen, H. M. L. Faulkner, M. P. Oxley, and D. Paganin, Phase retrieval and aberration correction in the presence of vortices in high-resolution transmission electron microscopy, Ultramicroscopy 88, 85 (2001).

[55] J. W. Goodman, Introduction to Fourier Optics (Roberts and Company, Englewood, CO, 2005).

[56] A. Mourka, J. Baumgartl, C. Shanor, K. Dholakia, and E. M. Wright, Visualization of the birth of an optical vortex using diffraction from a triangular aperture, Opt. Express 19, 5760 (2011).

[57] A. Mourka, Probing the Modal Characteristics of Novel Beam Shapes, Ph.D. thesis, University of St. Andrews, 2014.

[58] D. L. Andrews and M. Babiker, The Angular Momentum of Light (Cambridge University Press, Cambridge, UK, 2012).

[59] T. Niermann, J. Verbeeck, and M. Lehmann, Creating arrays of electron vortices, Ultramicroscopy 136, 165 (2014).

[60] M.-Á. García-March, A. Ferrando, M. Zacarés, J. Vijande, and L. D. Carr, Angular pseudomomentum theory for the generalized nonlinear Schrödinger equation in discrete rotational symmetry media, Physica D: Nonlin. Phenom. 238, 1432 (2009).

[61] G. Guzzinati, P. Schattschneider, K. Y. Bliokh, F. Nori, and J. Verbeeck, Observation of the Larmor and Gouy Rotations with Electron Vortex Beams, Phys. Rev. Lett. 110, 093601 (2013).

[62] M. V. Berry, Optical vortices evolving from helicoidal integer and fractional phase steps, J. Opt. A: Pure Appl. Opt. 6, 259 (2004).

[63] J. J. Healy and J. T. Sheridan, Sampling and discretization of the linear canonical transform, Signal Process. 89, 641 (2009).

[64] J. Masajada and B. Dubik, Optical vortex generation by three plane wave interference, Opt. Commun. 198, 21 (2001).

[65] K. O'Holleran, M. J. Padgett, and M. R. Dennis, Topology of optical vortex lines formed by the interference of three, four, and five plane waves, Opt. Express 14, 3039 (2006).

[66] A. Ferrando and M. A. García-March, Theory for the control of dark rays by means of discrete symmetry diffractive elements, J. Opt. 15, 044014 (2013).

[67] K. O‘Holleran, M. R. Dennis, and M. J. Padgett, Topology of Light's Darkness, Phys. Rev. Lett. 102, 143902 (2009).

[68] M. R. Dennis, R. P. King, B. Jack, K. O’Holleran, and M. J. Padgett, Isolated optical vortex knots, Nature Phys. 6, 118 (2010). 